\title{
Genome-wide suppressor screen identifies USP35/USP38 as therapeutic candidates for ciliopathies
}

\author{
I-Chun Tsai, ${ }^{1}$ Kevin A. Adams, ${ }^{1}$ Joyce A. Tzeng, ${ }^{1}$ Omar Shennib, ${ }^{1}$ Perciliz L. Tan, ${ }^{1,2}$ \\ and Nicholas Katsanis ${ }^{1,3,4}$ \\ ${ }^{1}$ Center for Human Disease Modeling, Duke University School of Medicine, Durham, North Carolina, USA. ${ }^{2}$ Rescindo \\ Therapeutics, Durham, North Carolina, USA. ${ }^{3}$ Stanley Manne Children's Research Institute, Ann \& Robert H. Lurie \\ Children's Hospital of Chicago, Chicago, Illinois, USA. ${ }^{4}$ Departments of Pediatrics and Cellular and Molecular Biology, \\ Northwestern University Feinberg School of Medicine, Chicago, Illinois, USA.
}

\begin{abstract}
The ciliopathies are a group of phenotypically overlapping disorders caused by structural or functional defects in the primary cilium. Although disruption of numerous signaling pathways and cellular trafficking events have been implicated in ciliary pathology, treatment options for affected individuals remain limited. Here, we performed a genome-wide RNAi (RNA interference) screen to identify genetic suppressors of BBS4, one of the genes mutated in Bardet-Biedl syndrome (BBS). We discovered 10 genes that, when silenced, ameliorate BBS4-dependent pathology. One of these encodes USP35, a negative regulator of the ubiquitin proteasome system, suggesting that inhibition of a deubiquitinase, and subsequent facilitation of the clearance of signaling components, might ameliorate BBS-relevant phenotypes. Testing of this hypothesis in transient and stable zebrafish genetic models showed this posit to be true; suppression or ablation of usp35 ameliorated hallmark ciliopathy defects including impaired convergent extension (CE), renal tubule convolution, and retinal degeneration with concomitant clearance of effectors such as $\beta$-catenin and rhodopsin. Together, our findings reinforce a direct link between proteasome-dependent degradation and ciliopathies and suggest that augmentation of this system might offer a rational path to novel therapeutic modalities.
\end{abstract}

Conflict of interest: NK and ICT are paid consultants of Rescindo Therapeutics Inc. NK also holds founder stock of the same commercial entity. PLT was a paid employee of Rescindo Therapeutics Inc. at the time of this research.

Copyright: (c) 2019, American Society for Clinical Investigation.

Submitted: May 21, 2019

Accepted: October 9, 2019

Published: November 14, 2019.

Reference information: JCI Insight 2019;4(22):e130516.

https://doi.org/10.1172/jci.

insight.130516.

\section{Introduction}

Ciliopathies are a group of $>100$ molecularly heterogenous or clinically overlapping disorders caused by defects in the primary cilium and its anchoring structure, the basal body $(1,2)$. Since cilia are present on almost all vertebrate cell types (1), it is not surprising that the cilium modulates a broad range of tissue and cellular events, including development, homeostasis, and even cancer progression (2,3). Disruption of ciliary function has been shown to cause numerous human genetic disorders, including polycystic kidney disease (PKD), nephronophthisis (NPH), Bardet-Biedl syndrome (BBS), Meckel-Gruber syndrome (MKS), Orofaciodigital syndrome 1 (OFD1), Joubert syndrome (JBTS), Jeune syndrome (JATD), Senior-Loken syndrome (SLS), and Leber congenital amaurosis (LCA). Consistent with their common organellar defect, ciliopathies also share overlapping phenotypes, including retinal degeneration, polydactyly, cystic kidney disease, situs inversus, intellectual disability, cerebellar hypoplasia, hydrometrocolpos, obesity, and liver dysfunction (4-6).

Although improved sequencing technologies have facilitated an improved diagnostic rate, this group of disorders remains largely untreatable. At present, the only credible approach proposed has been gene

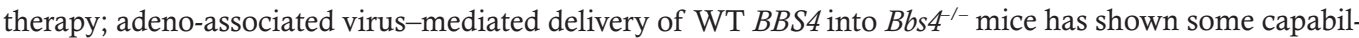
ity to prevent photoreceptor death and to preserve retinal function (7). However, with $>60$ ciliopathy loci identified to date (8), and most patients bearing private mutations, other modalities need to be developed.

In recent years, several loss-of-function mutations have been shown to confer protection from disease or from disease risk. For example, individuals with a CCR5 $\Delta 32$ mutation are resistant to CCR5-tropic HIV infection because of the lack of surface expression of HIV-entry receptor (9). Similarly, loss-of-function mutations in PCSK9 confer a lower level of low-density lipoprotein (LDL) and reduced risk of coronary heart disease (CHD) $(10,11)$. Likewise, Ezetimibe has been used to decrease plasma levels of LDL 
cholesterol by inhibiting the activity of the Niemann-Pick C1-like 1 (NPC1L1) protein (12); sequencing of a CHD cohort reported inactivating mutations in NPC1L1 associated with lower LDL levels and a lower risk of CHD (13). Finally, protein-truncating variants in SLC30A8 have been shown to reduce the risk of type 2 diabetes (14), encouraging the exploration of new therapeutic targets.

Because of such paradigms, suppressor screens have been proposed as a useful agnostic method to both understand disease pathomechanism and also to identify candidate genetic targets, around which novel therapeutics can be developed. For example, an ENU-mediated mutagenesis suppressor screen in Mecp2null mice, the gene responsible for Rett syndrome, identified a loss-of-function mutation in squalene epoxidase that ameliorates the symptoms caused by loss of Mecp2 (15). Additionally, a genome-wide screen in yeast found that knockdown of Dbr1, an RNA debranching enzyme, can alleviate the toxicity of TDP43 aggregates in Amyotrophic Lateral Sclerosis (16). More recently, a genome-wide CRISPR/Cas9 deletion screen uncovered several suppressors whose products can prevent cell death from the toxicity of dipeptide-repeat (DPR) protein (17).

An prominent role of primary cilium is to act as a signaling center $(18,19)$, facilitating the communication between extra- and intracellular signaling effector molecules during development and disease prognosis (2). To date, this organelle has been linked to several paracrine pathways crucial for development, including Wnt, Shh, and Notch (20). We posited that the identification of genes whose suppression could rescue aberrant signal transduction caused by ciliary dysfunction might have therapeutic value. To identify such targets, we designed a genome-wide RNAi screening paradigm, with the aim of isolating genes whose suppression can rescue hyperactive Wnt/ $\beta$-catenin ( $\beta$-cat) signaling in the absence of BBS4 (a ciliary gene mutated in patients with BBS, an archetypal ciliopathy; refs. 21, 22).

Here, we report the findings of our screen, in which 10 of 29 in vitro hits were found to rescue bbs4-dependent convergent extension (CE) defects in vivo. Considering the druggable potential and our previous finding that suggests the perturbation of proteasome-mediated protein degradation, we focused on USP35, a negative regulator of ubiquitin-proteasome protein degradation. Deeper characterization of USP35 (and its orthologue USP38) showed that suppression or ablation in vivo rescued a host of ciliopathy-associated anatomical pathologies, with concomitant rescue of the overabundance of key signaling molecules. These data further support a central role for the ubiquitin proteasome system (UPS) in the pathogenesis of ciliopathies and suggest that targeting this pathway via inhibiting USP35/USP38 deubiquitinase provides a rational route for the development of novel therapeutic modalities.

\section{Results}

Genome-wide suppressor screen identifies genes that reduce aberrant Wnt signaling caused by loss of BBS4. We reported previously that loss of $B B S 4$ expression leads to the hyperactivation of $\mathrm{Wnt} / \beta$-cat signaling

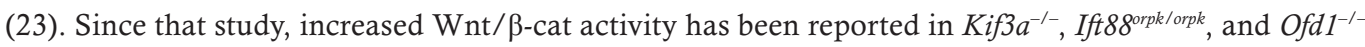
mice (24). Importantly, the canonical Wnt activation phenotype could be rescued by WT BBS4 and be recapitulated quantitatively in vivo (23). Therefore, we used this assay to design and execute a genomewide RNAi suppressor screen. We first generated a human retinal pigmented epithelium (RPE) cell line that stably expresses an shRNA against BBS4 (20) and a luciferase reporter with 8 concatenated T cell factor (TCF) binding sites (Figure 1A and Supplemental Figure 1; supplemental material available online with this article; https://doi.org/10.1172/jci.insight.130516DS1), whose luciferase activity will be elevated when increased $\beta$-cat binds to TCF and turns on the expression of luciferase. Cells selected clonally for reproducibility and dynamic range (Supplemental Figure 1) were then transfected with the Qiagen human whole-genome siRNA library, targeting about 22,000 genes. To improve the reproducibility of the screen and to reduce the false positive/false negative rate, the library was designed to contain 2 half libraries, in which 4 nonrelated siRNAs (2 siRNAs in each half library) target 1 gene (25). At 72 hours after transfection, cells were stimulated with WNT3a and collected for luciferase and lactate dehydrogenase (LDH) assays (for cell viability; Figure 1A). With the expectation that some siRNAs may target genes that influence cell viability, leading to false positives, luciferase readouts were normalized to LDH activity. Through this platform, we identified 29 genes that can reduce significantly $(\mathrm{z}<-3 ; P<0.05$, replicated) the hyperactivation of $\mathrm{Wnt} / \beta$-cat signaling in replicate wells (Figure $1 \mathrm{C}$ ).

AXIN2 expression is a direct target of $\mathrm{Wnt} / \beta$-cat signaling (26) and has been used to evaluate the activation of $\mathrm{Wnt} / \beta$-cat signaling (27). Consistent with the Wnt-reporter assay, BBS4 depletion enhances $A X I N 2$ expression significantly (Supplemental Figure 2). Therefore, to validate the 29 hits, we transfected 
siRNA of each of the 29 genes into the same cell type used for the primary screen and performed quantitative PCR (qPCR) to quantify AXIN2 message. In the context of BBS4 knockdown, suppression of 14 of 29 genes led to significant reduction of AXIN2 message in comparison with control siRNA (Figure 1B). Although the roles of some of these genes are unclear, the identified hits are involved in different cellular mechanisms (Supplemental Table 1). These results suggest the link of ciliopathies to both existing and potentially new cellular mechanisms. Given that these hits are identified through in vitro Wnt/ $\beta$-cat reporter, we next asked whether the rescue of hyperactive Wnt/ $\beta$-cat signaling can also be observed in vivo. During early development, hyperactivation of the Wnt/ $\beta$-cat pathway perturbs planar cell polarity, impairing proper CE $(23,28)$. Therefore, we investigated the in vivo effects of the 14 candidate genes by assessing their effects on the CE phenotype observed in bbs4 zebrafish morphants $(20,23)$. We designed morpholinos (MO) targeting 11 of the 14 candidates (DTX1, PITPNM2, ENTPD6, C14orf166B, TEX36, ZIC1, PTMA, ENGASE, ENPP7, TDRD12, and USP35) and guide RNA targeting 2 candidates (DRD5 and $P C F 11$; lack of appropriate splicing blocker site) to perturb the expression of candidate genes (RHOXF1 was excluded from further analysis due to the lack of a zebrafish ortholog) (Supplemental Figure 3). We scored the phenotypic effects resulting from suppression of these 13 candidates by $\mathrm{CE}$ analysis; we found that suppression of 10 of 13 rescued significantly the CE defects of bbs 4 morphants (Figure 1C, Figure 2A, and Supplemental Figure 4).

In vivo assessment of rescue efficacy by USP35 suppression. We and others have shown previously the existence of a functional relationship between ciliopathy proteins and the UPS (20, 23, 29). Importantly, prior in vitro studies motivated by protein-to-protein interaction between BBS4 and RPN10 showed that ciliary deficits can abolish proteasome-dependent protein degradation. Moreover, UPS-mediated protein degradation is also necessary for regulating multiple signaling pathways (30). Among our 10 candidate genes, we noted USP35, a ubiquitin-specific peptidase. Human USP35 belongs to the USP family of deubiquitinases and has been reported in the context of several cellular mechanisms, including the regulation of Park2-mediated mitophagy and Aurora B stability $(31,32)$. USP35 contains 2 USP catalytic domains that are conserved with USP38 (Supplemental Figure 5A), suggesting that USP35 and USP38 may play similar physiological roles. The orthologous roles of USP35 and USP38 are supported further by the fact that some species only have either USP35 (e.g., D. melanogaster) or USP38 (e.g., D. rerio) (33). Given these observations, we tested the rescue effects of each of USP35 and USP38 in human cells and usp38 in zebrafish. As a first test, we used a BBS4-depleted RPE cell line and transfected it with siRNA targeted to either USP35 or USP38, followed by assessment of their respective ability to ameliorate the hyperactivation of Wnt/ $\beta$-cat (Supplemental Figure 5, B and C). Suppression of either USP35 or USP38 attenuated AXIN2 expression (Supplemental Figure 5D), with USP38 having a more modest effect, likely explaining why this molecule was not detected in our primary screen. Cosilencing of both USP genes led to a more significant reduction of AXIN2 expression than either transcript alone, suggesting overlapping functions (Supplemental Figure 5D).

The zebrafish genome has a sole copy of usp38 with no detectable usp35 ortholog. We therefore generated both transient (morphants, MOs) and genetic (CRISPR/Cas9) models (Supplemental Figure 6 and Methods). We then asked whether suppression or deletion of usp 38 could promote proteasome-dependent protein degradation, facilitating the clearance of signaling molecules and rescue of developmental phenotypes. As a first test, we asked whether suppression of usp38 can rescue CE defects of bbs 4 morphants $(20,23,34)$. To obtain quantitative data for this experiment, we measured the angle between the tip of the head and the tail of 8-10 somite embryos, a measurement that captures body length in early embryos (23), and binned them into 3 categories: normal (under 2 SDs from control); Class I (2-4 SDs from control) and Class II (>4 SDs from control).

While suppression of bbs4 led to approximately $80 \%$ embryos with CE defects, coinjection with usp38-MO reduced the incidence of pathology and the severity of the phenotype (25\% Class I and $15 \%$ Class II compared with 35\% Class I and 45\% Class II in bbs 4 morphants; $P<0.0001$; Figure 2A). This observation indicates that in vivo suppression of usp38 can ameliorate the hyperactivation of the Wnt/ $\beta$ cat pathway, consistent with our in vitro screen.

A common feature of many ciliopathy patients is abnormal renal morphology, including but not limited to calyceal malformation, cyst formation, and fetal lobulation (35). Therefore, we next evaluated the effects usp35/usp38 suppression on renal development in 4-day postfertilization (dpf) zebrafish larvae. Consistent with other studies in ciliary morphants and mutants (36), bbs4 morphants displayed atrophy and 
A

A RPE cells
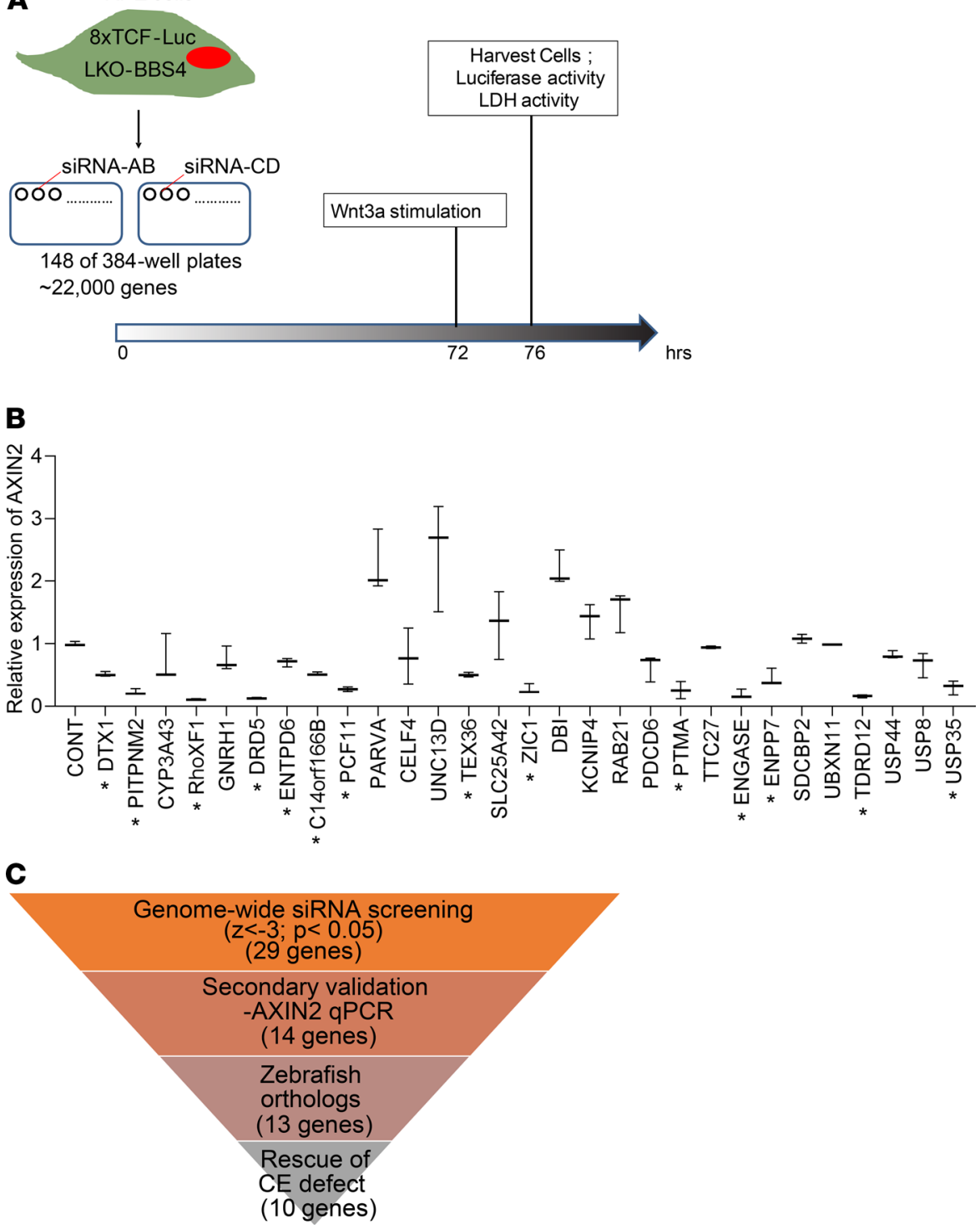

Figure 1. Genome-wide siRNA screening to identify the therapeutic candidate target for ciliopathies. (A) Experimental design of genome-wide siRNA screening. (B) AXIN2 qPCR was performed as the secondary validation. The same cell line used in the primary screen was transfected with siRNA targeting the 29 hits identified from the primary screening. Compared with the control-siRNA, relative expression level of AXIN2 from triplicated experiments is presented in the box-andwhiskers plot (Tukey's post hoc test). Asterisks denote the genes that reduce AXIN2 expression significantly $(P<0.05$; 2 -tailed Student's $t$ test). (C) Chart of the results from primary screening, secondary validation, and in vivo CE assays.

diminished convolution in proximal renal tubules $(\sim 65 \%)$, a phenotype reminiscent of renal immaturity of BBS patients and 1 possible source of cysts (ref. 37 and Figure 2B). In line with our CE studies, suppression of usp 38 improved - but did not fully rescue - the renal abnormalities of $b b s 4$ morphants by about $20 \%$ (Figure 2B). Since a functional assay would be more straightforward to statistically assess the rescue efficacy, we performed extensive experiments to develop a proteinuria assay in our zebrafish models. However, likely due to (a) the detection limitation and (b) high assay variability, this experiment was not possible in a zebrafish system. A murine model will be required to address this aspect of the work.

The use of MOs to target genes of interest is more homogenous and less time-consuming but can be confounded by off-target effects (38). To test the specificity of our MO-induced findings, we implemented a CRISPR/Cas9 genome-editing approach. Guide RNAs targeting exon 3 of bbs 4 and exon 1 of usp38 induced efficient ( 99\%) Cas9-mediated genome editing (Supplemental Figure 6). Expression of human BBS4 and USP35 mRNA in the bbs4 gRNA/Cas9 and usp38 gRNA/Cas9 significantly rescue the phenotypic defects, 
A

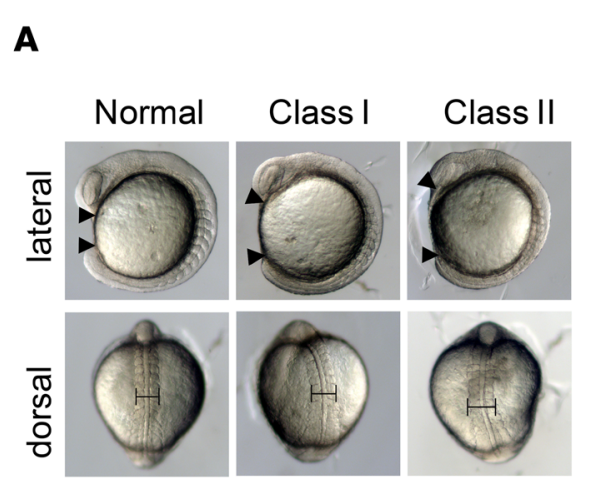

B

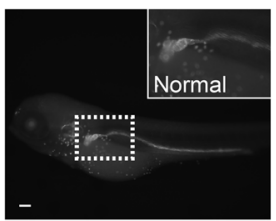

control

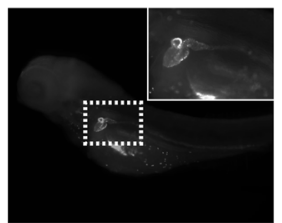

bbs4-MO

C

usp38-MO

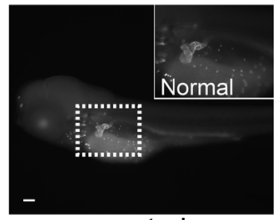

control

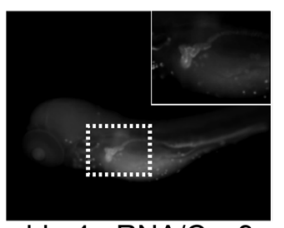

bbs4-gRNA/Cas9 usp38-gRNA/Cas9

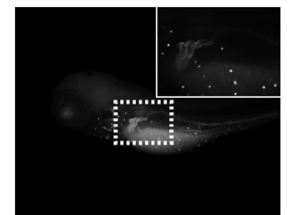

usp38-MO
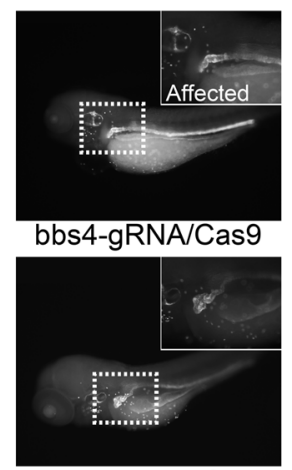

usp38-gRNA/Cas9

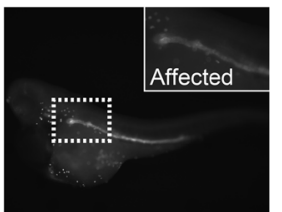

bbs4-MO

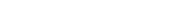

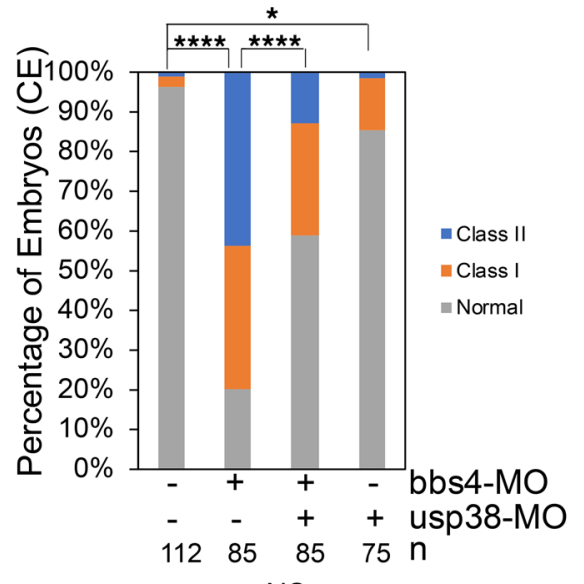
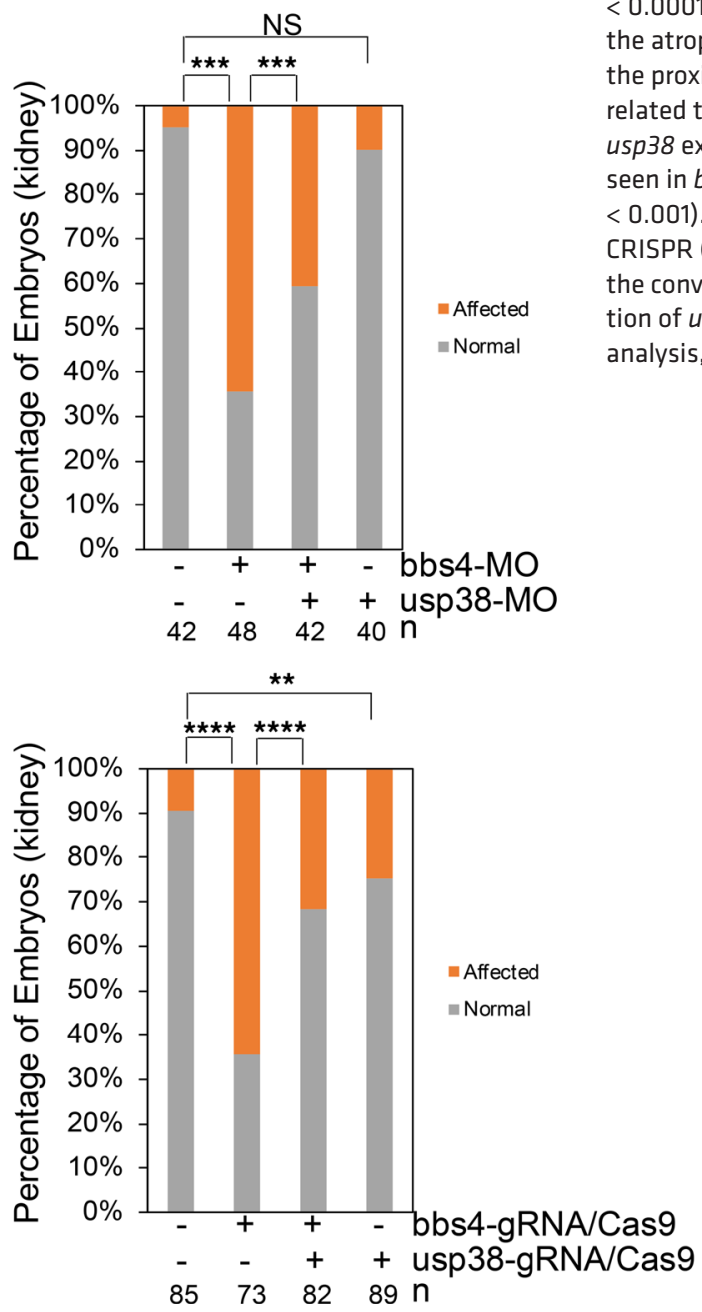

Figure 2. Suppression of usp38 (USP35 ortholog in zebrafish) ameliorates the defects caused by depletion of bbs4. (A) Morpholinos were injected as described in the Methods. Depletion of bbs4 results in CE defects, including wider anterior-posterior body gap, somite, and loss of eyes (mostly in Class II). To quantitatively determine the CE phenotype, the angle of body gap was measured in each embryo, and the embryos were classified as described in Methods. Coinjection of usp38-MO reduces both Class I and Class II embryos. Arrowheads represent the tip of anterior and posterior body axis. Brackets represent the width of somite. $\left(\chi^{2}\right.$ analysis, ${ }^{*} P<0.05,{ }^{* * * *} P$ $<0.0001$ ). (B) Depletion of bbs4 leads to the atrophy and deficient convolution in the proximal tubules, a phenotype that is related to cyst formation. Knockdown of usp38 expression ameliorates renal defects seen in bbs 4 morphants ( $\chi^{2}$ analysis, ${ }^{* *} P$ $<0.001)$. Scale bar: $100 \mu \mathrm{m}$. (C) While bbs 4 CRISPR (mosaic) mutant exhibit defects in the convolution of proximal tubules, deletion of $u$ sp38 ameliorates these defects $\left(\chi^{2}\right.$ analysis, ${ }^{* *} P<0.01,{ }^{* * *} P<0.0001$ ). 
we see a milder effect by CRISPR than MO, which suppressed more uniformly. We used both approaches in this study. In our view, the focus is not the magnitude of rescue efficacy of both techniques but the direction of effect. Together, the results by using both approaches suggest that suppression of usp 38 can ameliorate the defects of BBS suppression or ablation in zebrafish.

Exploring the molecular mechanism of USP35 suppression mediated rescue. We next probed the molecular basis of the observed rescue using our in vitro and in vivo tools. Specifically, we asked whether USP35/ USP38 regulates the stability or deubiquitination of signaling components known to be perturbed by bbs 4 depletion, such as the Wnt/ $\beta$-cat pathway.

First, we returned to the in vitro model. We have shown previously that suppression of BBS4 leads to accumulation of $\beta$-cat in the HEK293 cells (23). Therefore, we cultured BBS4-depleted HEK293 cells, pulled down endogenous $\beta$-cat by immunoprecipitation, and assessed the effects of USP35 suppression on its ubiquitination. We found that the ubiquitination of $\beta$-cat increases in cells depleted of USP35 (Figure 3A), suggesting that suppression of USP35 will facilitate the degradation of $\beta$-cat. Next, we asked whether USP35 also affects the stability of other ciliopathy-relevant signaling pathways, such as Notch. We have reported previously that depletion of bbs 4 in the HEK293 cells leads to accumulation of Notch intracellular domain (NICD), a key component of Notch signaling (20). We reproduced the previous finding, with BBS4 suppression causing the accumulation of NICD in our cell-based model (Supplemental Figure 9 and Figure 3B). Consistent with a candidate rescue mechanism, cosuppression of USP35 with BBS4 facilitated the degradation of NICD (Figure 3B), suggesting that improved degradation of aberrantly accumulated proteins is a strong candidate rescue mechanism.

Hedgehog $(\mathrm{HH})$ signaling is also a major pathway regulated by both the primary cilium and the proteasome (40). We therefore asked whether disruption of USP35 affects $\mathrm{HH}$ signaling transduction. $\mathrm{HH}$ signaling plays important roles during retina differentiation, as well (41). To assess the activation of $\mathrm{HH}$, we perform qPCR in RPE cells for PTCH1 and BCL2, whose expression increases when $\mathrm{HH}$ is activated $(42,43)$. We found that the expression level of PTCH1 and BCL2 were elevated in BBS4-depleted RPE cells compared with nonsilencing control cells (7- and 3-fold of increase, respectively; Supplemental Figure10A and 10B). However, knockdown of USP35 attenuated this phenotype (Supplemental Figure 10, A and B). Although which HH component might be targeted by USP35 is unknown, these results suggest that USP35 suppression in not restricted to Wnt-mediated defects.

Next, we asked if the deubiquitination function of USP35 is involved in the rescue mechanism of USP35, as opposed to hitherto unknown functions of this molecule. For this experiment, we generated a dominant negative mutant of USP35, in which Cys450 is mutated to alanine, blocking its activity to cleave the ubiquitin chain $(31,33)$. If deubiquitinase activity of USP35 is required for rescue, expression of USP35 (C450A) should serve as a USP35 inhibitor and ameliorate the defects of bbs4 MOs in zebrafish. To test this notion, we coinjected bbs4 MO with either WT or dominant-negative human USP35 mRNA. We found that overexpression of human USP35 (C450A) encoded message ameliorated significantly both CE and renal convolution defects caused by bbs 4 depletion $(P<0.01$, Figure $3 C ; P<0.05$, Figure $3, \mathrm{D}$ and $\mathrm{E})$. This rescue effect was specific to USP35 (C450A); expression of WT USP35 had no effect (Figure 3, C, D, and E). These data suggest that the deubiquitinase function of USP35 is the likely driver of $b b s 4$ rescue.

Given our observations, we wondered whether this enzymatic activity was specific to USP35 or whether other deubiquitinases could also be efficacious. To address this question, we reanalyzed our suppression screen data for all known deubiquitinases (Supplemental Table 2). Suppression of some deubiquitinases enhanced (z-score $>2$ ); some had no effect (z-score between -2 and 2); and some decreased (z-score $<-2)$ the hyperactivation of $\mathrm{Wnt} / \beta$-cat signaling. To investigate further the specificity of our in vitro and in vivo assays, we asked whether a deubiquitinase not able to rescue BBS4-mediated Wnt/ $\beta$-cat hyperactivation in vitro can rescue bbs 4 morphant pathology in zebrafish. Recently, a small molecule inhibitor for USP14, IU1, has been reported; treatment of IU1 induces the degradation of proteasome substrate $(44,45)$. Since our suppressor screen showed that BBS4-mediated Wnt/ $\beta$-cat hyperactivation cannot be rescued by USP14 depletion, we tested the effect of usp 14 knockdown in bbs 4 morphants. Cosuppression of usp 14 did not rescue the CE and renal defects in bbs4 morphants (Supplemental Figure 11, A, B, and C), while treatment of IU1 also cannot rescue $b b s 4$ morphant (Supplemental Figure 11D) This result suggests the consistency and specificity between the cell-based screen and animal modeling assays.

Taken together, our results suggest that: (a) depletion of bbs 4 causes the aberrant accumulation of signaling components; (b) suppression of USP35 can enhance the ubiquitination and facilitate protein clearance; and (c) the rescue mechanism likely operates through the inhibition of the deubiquitinase function of USP35. 

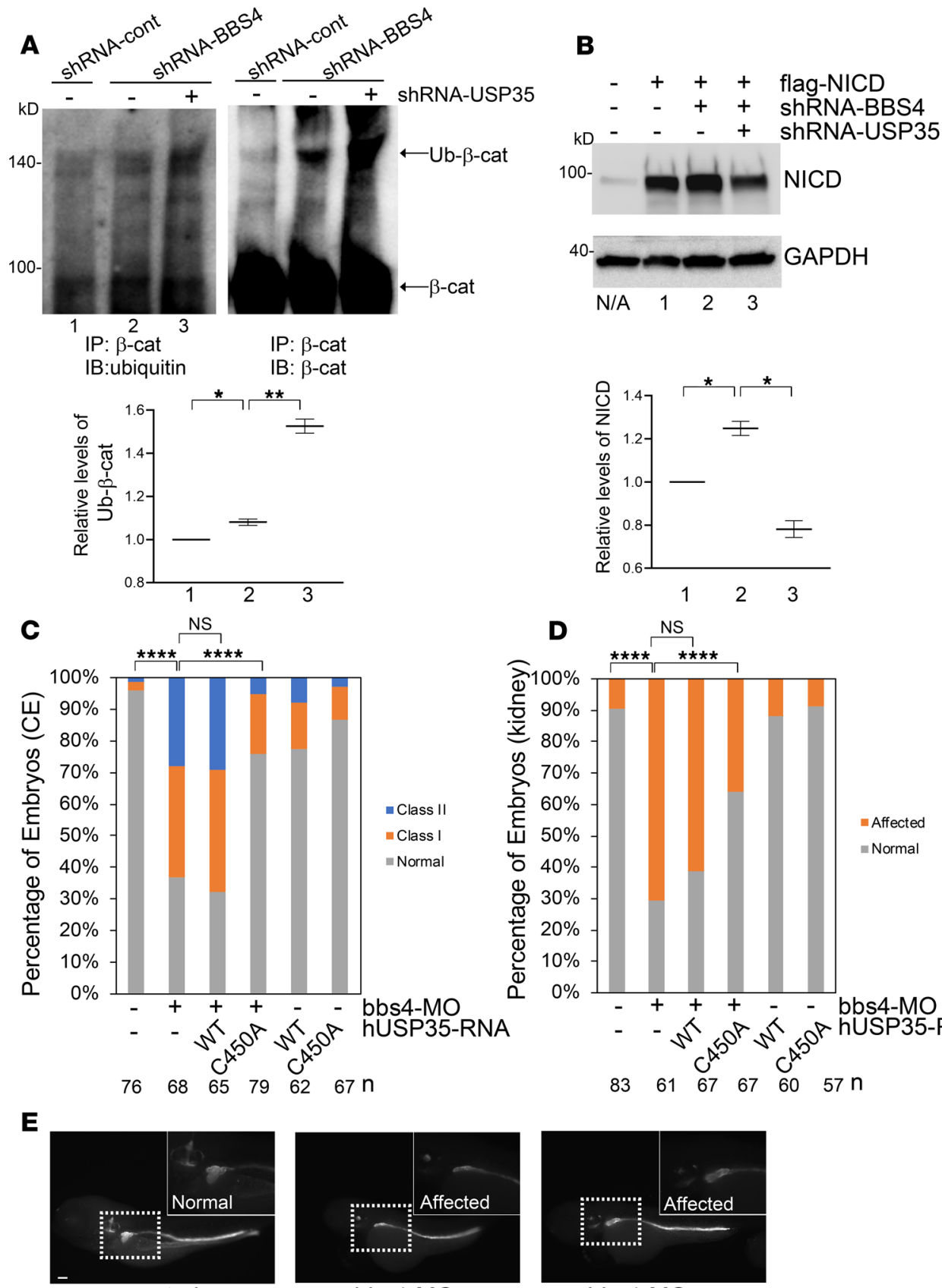

control

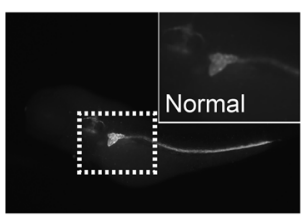

bbs4-MO + hUSP35(C450A)

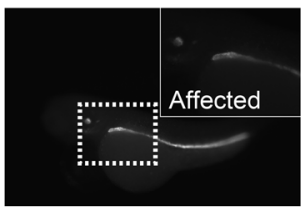

bbs4-MO

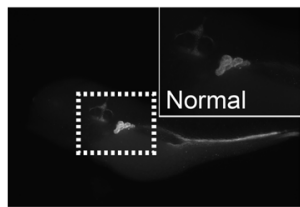

hUSP35(WT)
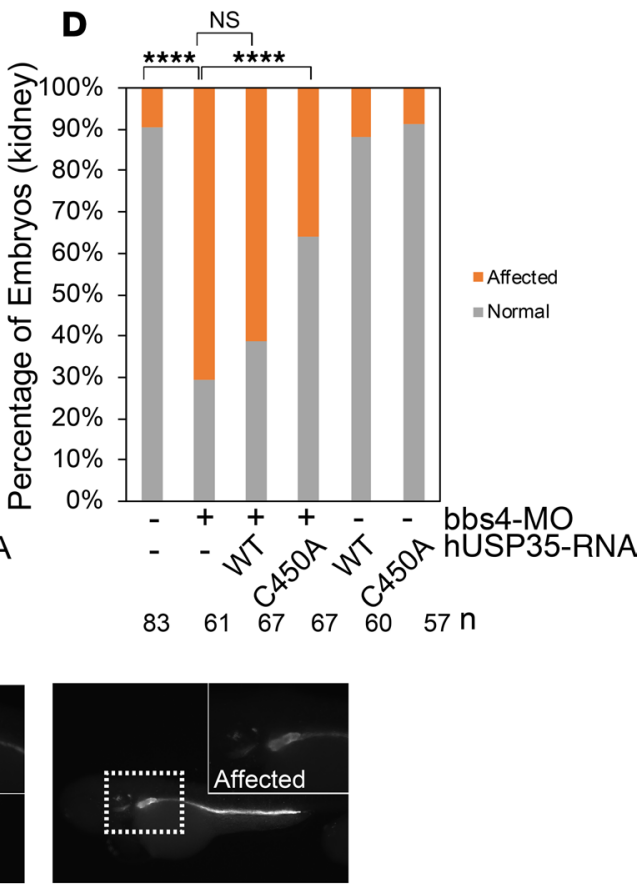

bbs4-MO +

hUSP35(WT)

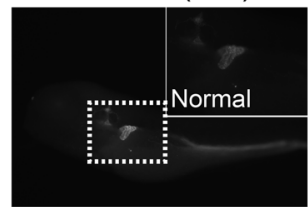

hUSP35(C450A)
Figure 3. The molecular mechanism by which USP35 suppression ameliorate the defects caused by BBS4 depletion. (A) Suppression of USP35 enhances the ubiquitination of $\beta$-catenin. BBS4-depleted HEK293 cells were transfected with either shRNA-control or shRNA targeting to USP35. 72 hours after transfection, immunoprecipitation of $\beta$-catenin was performed, followed by immunoblotting with $\beta$-catenin and ubiquitin. The relative quantification of $\beta$-catenin ubiquitination was performed with QuantityOne. ${ }^{*} P<0.05$, ${ }^{* *} P<0.01$ (2-tailed Student's $t$ test).

(B) USP35 suppression facilitated the degradation of accumulated NICD. HEK293 cells were transfected with the plasmids as indicated in the figure. Seventy-two hours after transfection, cells were harvested and subjected to immunoblotting of NICD and GAPDH (loading control). Relative levels of NICD from 2 experiments are indicated in the bottom of the panel. ${ }^{*} P<0.05$ (2-tailed Student's $t$ test) (C) Coexpression of dominant negative human USP35 in bbs4 morphants ameliorates CE defects. Zebrafish embryos were injected with bbs4 morpholino and mRNA encoding either WT or dominant negative human USP35. CE defects can be ameliorated by coexpression of hUSP35 (C450A) but not hUSP35 (WT). ${ }^{* * *} P<0.0001\left(\chi^{2}\right.$ analysis). (D) Loss of bbs4 leads to the atrophy and deficit convolution in the proximal tubule. Coexpression of hUSP35 (C450A) but not hUSP35 (WT) can ameliorate the renal development defects. ${ }^{* * *} P<0.0001\left(\chi^{2}\right.$ analysis). (E) Represented images of D. Scale bar: $100 \mu \mathrm{m}$.

Investigating the efficacy of USP35 suppression/ablation in retinal degeneration. Our screening and validation data, together with prior studies that implicated proteasomal function in the pathomechanism of ciliopathies $(20,23)$, suggested that suppression of USP35 might offer a rational therapeutic route. However, our data measured efficacy in systems whose therapeutic implementation would not be useful because they affect early developmental processes. We thus turned our attention to retinal degeneration, a postnatal, progressive phenotype pathognomonic of BBS (46-49) and other ciliopathies (50-52). 

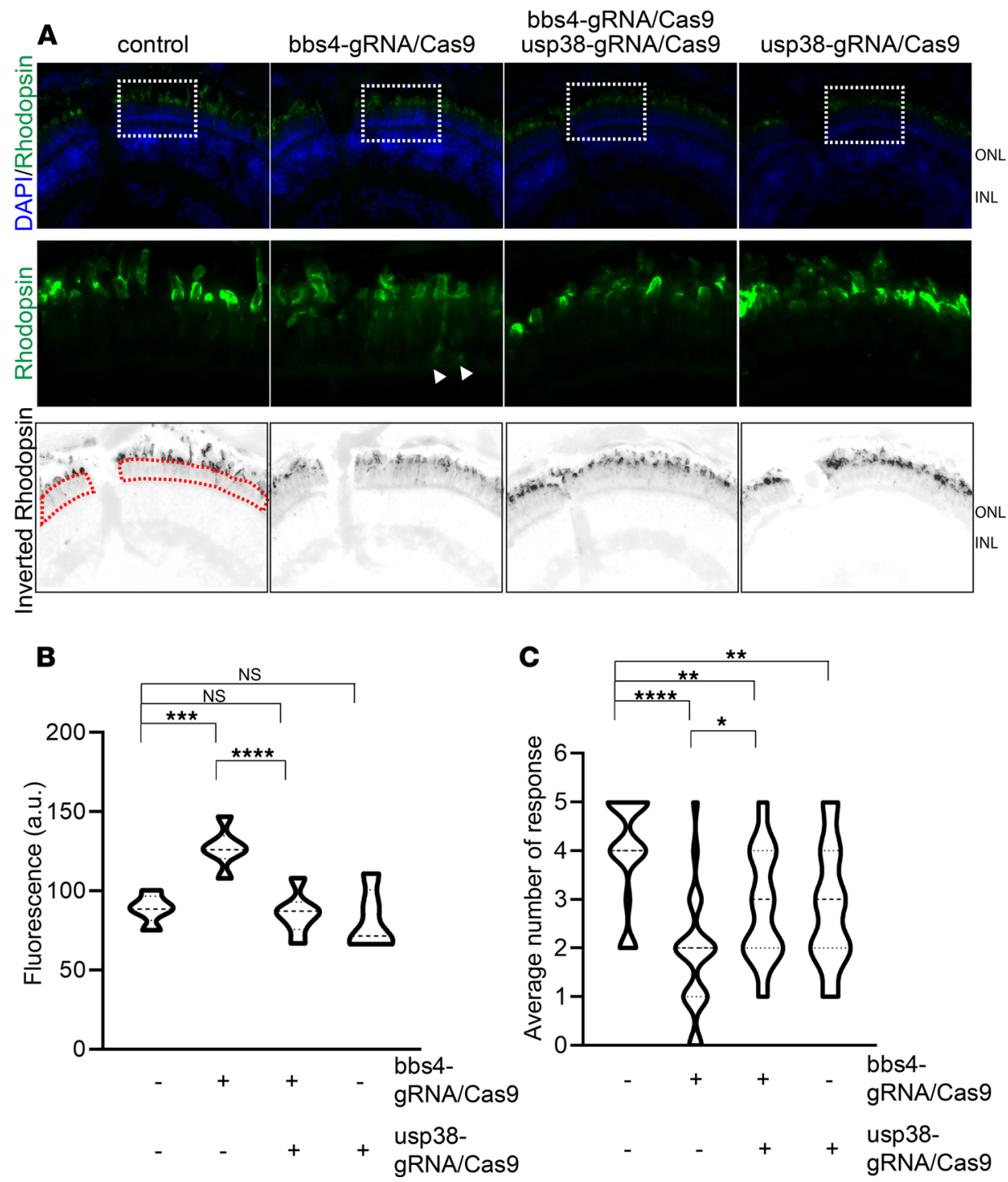

Figure 4. Rescue of the bbs4 CRISPR mutant phenotype with coablation of usp38 in 5dpf zebrafish larvae. (A) Mislocalization of rhodopsin staining (green) is trapped in the cytoplasm of the outer nuclear layer (ONL) cells in bbs 4 mutants (arrowheads), but localization is restored to normal in double bbs4/usp38 mutants. Blue, DAPI. Top- and bottom panels of images were taken at 20x; midpanel of images were taken at 40x. (B) The rhodopsin located in the cytoplasmic region (indicated by dashed red line in $\mathbf{A}$ ) of ONL cells was quantified by Image) (NIH). Ablation of bbs 4 causes a significant increase of rhodopsin mislocalization. Coablation of usp38 rescues this defect (1-way ANOVA). ${ }^{* *} P<0.001$; ${ }^{* * *} P<0.0001$. (C) Assessment of visual function by visual startle response (VSR). Loss of bbs4 leads to deficient startle response upon light stimulation. However, the visual ability can be improved by coablation of usp38 (1-way ANOVA). ${ }^{*} P<0.05$; ${ }^{* *} P<0.01$; ${ }^{* * *} P<0.0001$.

Mislocalization of rhodopsin in the inner segment is a hallmark of $B b s 4^{-1-}$ retinas, a molecular pathology also associated with the progression of retinal degeneration $(7,53)$. A recent report suggests that enhanced proteasome activity might be a therapeutic target for dominant retinal degeneration caused the mislocalization of the p.Pro23His rhodopsin mutant (54). Therefore, we considered mislocalization of rhodopsin as an attractive target, especially since accumulation of unstable GFP, a reporter of proteasome activity, was also reported in a $B b s 4^{-{ }^{-}}$mouse mutant (20). We examined the retinas of $5 \mathrm{dpf}$ zebrafish larvae and assessed whether mislocalization of rhodopsin can be detected in a bbs4 CRISPR/Cas9 model. We generated retinal sections, stained them with an anti-rhodopsin antibody, and quantified the amount of rhodopsin in the cytoplasm of the outer nuclear layer (ONL). Compared with WT embryos, bbs4 CRISPR mutants (>99\% mosaicism, Supplemental Figure 6) had 
significantly increased rhodopsin mislocalization $(P<0.01$; Figure $4, \mathrm{~A}$ and $\mathrm{B})$. In contrast, injection of CRISPR gRNAs targeting usp38 (>99\% mosaicism, Supplemental Figure 6) alone had no impact on retinal morphology. However, coinjection of bbs 4 and usp38 gRNAs with Cas 9 rescued rhodopsin mislocalization significantly ( $P<0.05$; Figure $4, \mathrm{~A}$ and $\mathrm{B})$.

As a second test, we sought to assess the impact of usp 38 depletion on visual function in bbs4-depleted models. We adapted the method described by Scott et al. to monitor startle activity of $5 \mathrm{dpf}$ larvae under light stimulus (55). Consistent with their IHC, while bbs4 CRISPR larvae had minimally recordable response to light stimulus compared with controls $(P<0.001$; Figure $4 \mathrm{C})$, codepletion of usp 38 and bbs 4 improved visual function, as indicated by the ability to respond to light stimulation ( $P$ $<0.05$; Figure 4C). This result suggests that ablation of usp38 can ameliorate the visual deficits of bbs 4 CRISPR larvae. Taken together, our results demonstrate the potential of usp 38 suppression to prevent mislocalization of rhodopsin and to ameliorate visual defects in bbs4 mutants.

Assessing the efficacy of USP35 suppression in other ciliopathies. We next asked whether USP35/USP38 suppression might also exert amelioration effects in other ciliopathy models. Intraflagellar transport 88 (IFT88) is a core protein of anterograde IFT complex, which is responsible for transporting protein cargo from basal body to the tip of cilia (1). Mutations of IFT88 are also associated with renal cysts and retina degeneration $(56,57)$. Therefore, we suppressed usp 38 in an ift88-depleted model and asked whether knockdown of usp 38 can ameliorate the phenotypic defects of ift88 morphants; similar to the bbs4-depleted model, suppression of ift88 also results in defects of CE movements (58). While knockdown of ift 88 led to $65 \%$ of embryos with CE phenotypes (Supplemental Figure 12; $P<0.0001$ compared with controls), cosuppression of usp38 in ift88 morphant significantly ameliorated the CE defects (Supplemental Figure 12; $P<0.0001$ ). We then evaluated the rescue efficacy of usp 38 knockdown in the retina of iff 88 morphants. We harvested retinas of 5 dpf embryos and performed immunostaining to measure, quantitatively, the abundance of rhodopsin in the inner segment. Similar to ift88 mutants (59), we observe mislocalized rhodopsin in the ONL of ift88 morphants (Figure 5, A and B; $P<0.0001$ ). While knockdown of usp38 alone does not cause significant impact regarding rhodopsin localization (Figure $5, \mathrm{~A}$ and $\mathrm{B}$ ), cosuppression of usp 38 in ift 88 morphant significantly ameliorated the mislocalization of rhodopsin (Figure 5, A and B; $P<0.01$ ). Finally, we evaluated the impact of usp 38 depletion on visual function in ift 88 morphants. Consistent with the results of rhodopsin immunostaining, ift88 morphant exhibits a reducing number of responses to light stimulation (Figure $5 \mathrm{C} ; P<0.0001$ ). This defect was ameliorated by the suppression of usp38 (Figure 5C; $P<0.0001$ ). Together, these results indicate that suppression of usp38 can ameliorate the phenotypic defects caused by loss of ift 88 , suggesting that the therapeutic potential of usp35/usp38 is not limited to BBS4.

\section{Discussion}

While the advancement of sequencing technologies has significantly improved the diagnostic rate of ciliopathy patients, the lack of available treatment options for managing disease progression remains challenging. To identify therapeutic targets, we and others have been motivated to understand the pathomechanism of disease, allowing for the elucidation of targets that can restore the dysfunction caused by the loss of ciliary genes $(2,20)$. Here, we capitalized on our previous findings that deficient ciliary function results in hyperactivation of Wnt/ $\beta$-cat signaling and asked which genes, when suppressed, can ameliorate of the aberrant Wnt/ $\beta$-cat hyperactivation. By performing genome-wide siRNA screening, we identified 10 genes that, when suppressed, can ameliorate the defects caused by loss of ciliary gene function. From a therapeutic standpoint, loss-of-function mutations in some of these loci have been associated with cellular or organismal pathology that renders them poor targets. For example, loss-of-function mutations in ZIC1 cause craniosynostosis (60), while suppression of PTMA, a caspase 9 regulator, renders cells sensitive to UV-mediated apoptosis (61). From a mechanistic, signaling perspective, however, the discovered suppressors potentially link BBS4 to both existing and new links of ciliary biology. The suppressor DTX1 encodes an E3 ubiquitin ligase, which is known to regulate Notch signaling through both ubiquitination-dependent and -independent mechanisms (62-64). In contrast, Engase is an $\mathrm{N}$-acetylglucosaminidase, supporting the recent observation that regulation of this posttranslational modification might be relevant to ciliary transport and function (65), whereas PITPNM2 is relevant to the function of the retinal degeneration B locus in Drosophila (66). We were surprised with the identification of DRD5 as a potential ameliorator, although the fact that DRD5 alone disrupts early development reduces its potential suitability as a therapeutic target (Supplemental Figure 4). Furthermore, we speculate that the ability of DRD5 and BBS4 suppression to correct early development might intimate that they have opposite roles. 

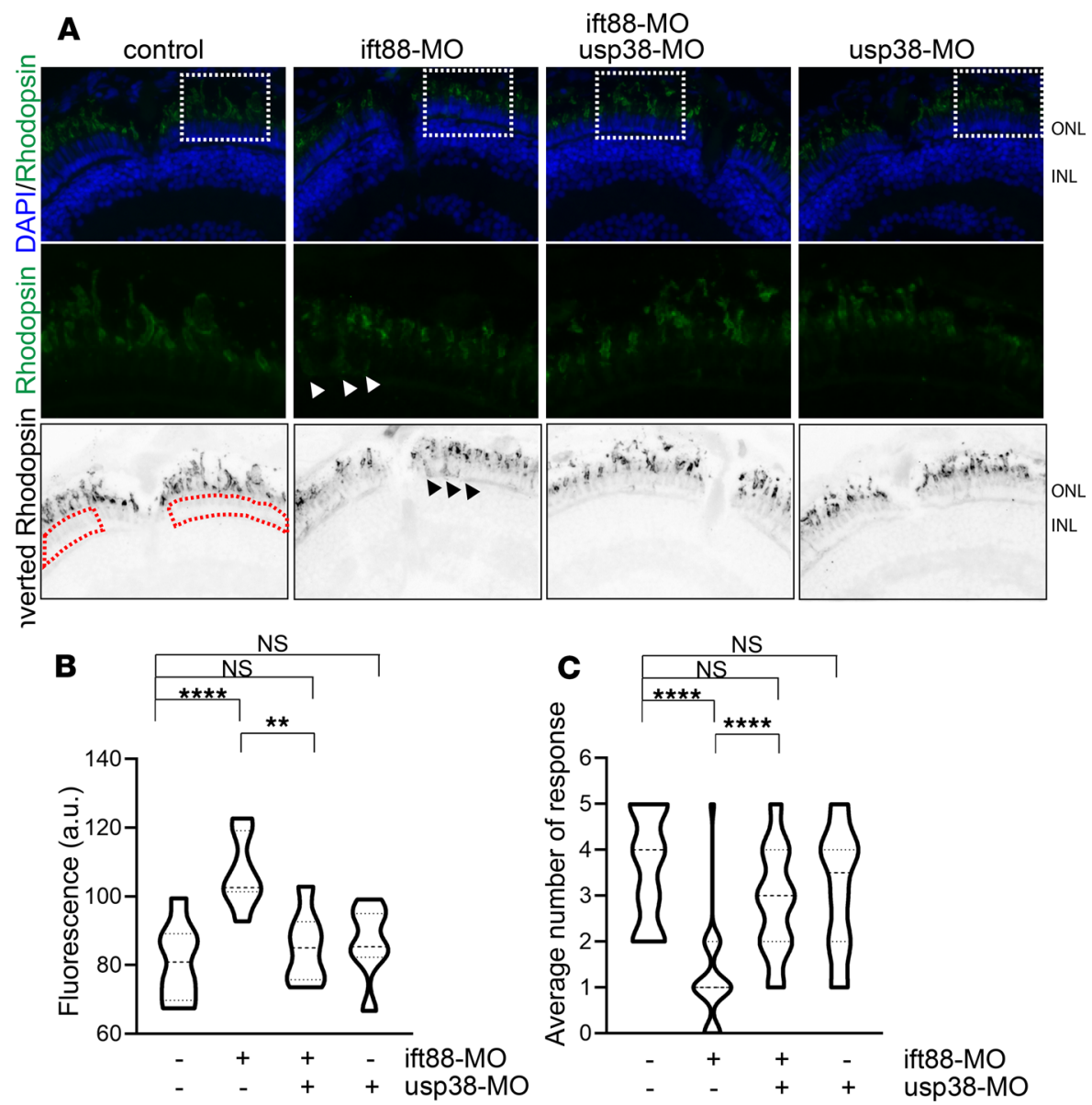

Figure 5. Rescue of the ift88 morphant phenotype with cosuppression of usp38 in $\mathbf{5 d p f}$ zebrafish larvae. (A) Mislocalization of rhodopsin staining (green) is trapped in the cytoplasm of the outer nuclear layer (ONL) cells in ift88 morphant (arrowheads), but localization is restored to normal in double bbs4/usp38 mutants. Blue, DAPI. Top and bottom panels of images were taken at 20x, and midpanel of images were taken at 40x. (B) The rhodopsin located in the cytoplasmic region (indicated by dashed red line in $\mathbf{A}$ ) of ONL cells was quantified by Image (NIH). Suppression of ift88 causes a significant increase of rhodopsin mislocalization. Cosuppression of usp38 rescues this defect (1-way ANOVA). ${ }^{* *} P<0.01 ;{ }^{* * *} P<0.0001$. (C) Assessment of visual function by visual startle response (VSR). Knockdown of ift88 leads to deficient startle response upon light stimulation. However, the visual ability can be improved by cosuppression of usp38 (1-way ANOVA). ${ }^{* * *} P<0.0001$.

Given our prior observations regarding the roles of proteasomal regulation in BBS $(20,23)$, we focused on USP35, whose function is to remove ubiquitin chains from targeted proteins, preventing their degradation by the proteasome $(30,33)$. UPS has been reported to regulate the biosynthesis of primary cilium (29), warranting the questions of (a) whether USP35 may play a role in ciliogenesis and (b) whether restoration of the functional mechanism of USP35 is cilium dependent. However, these questions do not exclude the possibility that USP35 may modulate signaling pathways through a cilium-independent mechanism because a low percentage of ciliated hTert-RPE1 ( 10\%) can be observed under normal culture condition (with $10 \%$ FBS in F12/DMEM). To date, downstream targets of USP35 remain elusive. Park et al. reported that USP35 regulates cell cycle progression by interacting with and deubiquitinating Aurora B, preventing its degradation. Suppression of USP35 significantly reduces the number of cells expressing phospho-histone H3, suggesting an inhibition of cell proliferation (31). Aberrant cell cycle progression has been linked as a mechanism of renal cyst formation (67-69); this, coupled to the reported USP35-mediated mitotic progression, may explain why USP35 suppression is able to ameliorate the renal defects observed in bbs 4 morphants. However, the observed rescue of gastrulation movements and rhodopsin trafficking, as well as the USP35-mediated degradation of $\beta$-cat, suggests that other USP35 targets likely drive the observed rescue. Similarly, in addition to Wnt/ $\beta$-cat signaling, other cilium-mediated pathways (such as Hh and Notch signaling; ref. 20, 70, 71) may be affected by USP35 suppression, as well. Although we have also observed that USP35 suppression affects 
Notch and $\mathrm{HH}$ signaling pathways, further proteomic analyses to identify targets of USP35/USP38 will likely be required to inform the biochemical mechanism of rescue.

Finally, we note that USP35 was not the only USP to rescue BBS4-dependent signaling defects. USP44 also significantly rescued these defects (Supplemental Table 2); it is a protein shown to regulate the ubiquitination of CDC20 (72), which in turn regulates ciliary length and disassembly (73). It will be important to understand what subset of deubiquitinases are relevant, directly or directly, to ciliary biogenesis, homeostasis, and function and what their role might be in conferring protection from the consequences of mutations that affect this organelle.

In summary, we have developed a functional screening platform to identify potential therapeutic targets for ciliopathy patients. In combination with previously established zebrafish models, we observed that suppression of the USP35 ortholog in zebrafish can restore the renal morphology and visual ability, 2 common features of ciliopathy patients. Further applying these discoveries, we speculate that inhibition of USP35 or other related molecules might be of therapeutic value. Importantly, suppression of USP35 or other similar treatments did not induce by themselves overt pathology in the zebrafish embryo, suggesting that treatment paradigms based on this molecule might be tolerated in humans.

\section{Methods}

Generation of cell lines and reporter plasmids. hTert-RPE1 (ATCC) cells were grown in DMEM and Ham's F-12 Nutrient 1:1 mixture (F12/DMEM, Invitrogen) with 10\% FBS and 2 mM L-glutamine. To generate the Wnt/ $\beta$-cat reporter, the TCF/LEF binding sites were excised from the SuperTOPFLASH construct (74) using $K p n I$ and HindIII and cloned into pGL4.18 [Luc2P/neo] vector (Promega). The hTert-RPE1_WntRep cell line was generated by FuGene6 transfection (Promega) with a Wnt reporter followed by selection with Geneticin (500 $\mathrm{g} / \mathrm{mL}$; Invitrogen). The short hairpin targeting BBS4 (5'-GGCACAAGACCAGTTGCACAA-3') was cloned into the pLKO vector (Addgene, 10878) and transduced into hTERT-RPE1_WntRep cells, followed by selection with puromycin (MilliporeSigma). Wnt3a conditioned media was obtained by collecting the cultured media from L-Wnt3a cells.

Genome-wide suppressor screen. Reverse transfection of siRNA (15 nM) was performed with RNAiMax reagent (Invitrogen). hTERT-RPE1_WntRep_shRNA-BBS4 cells $\left(4 \times 10^{3}\right.$ cells/well) were seeded into 384well plates containing siRNA and transfection reagents. At 72 hours after transfection, cells were stimulated with Wnt3a media and incubated for 4 hours. Cells were lysed with Passive Lysis Buffer $(25 \mu \mathrm{L} /$ well) (Promega). Luciferase and LDH activity of lysates were measured using the Luciferase Reporter Assay System (Promega) and LDH plus (Roche) on the FLUOstar Omega microplate reader (BMG Labtech), and they were analyzed with MARS Data Analysis Software (BMG Labtech). The luciferase results of each well were divided by the LDH results to account for cell viability for each knockdown condition. Since knockdown of BBS1 has been shown to activate Wnt/ $\beta$-cat signaling (23), BBS1 siRNA was utilized as a positive control. For quality control of the assay, we included 12 wells/plate of siRNA-control and BBS1 siRNA (5'-TTGCCCTTGTGTCTATGTGTAAA-3') to be able to calculate Z' (1-3 [SD $\left.\mathrm{BBS}_{1}+\mathrm{SD}_{\text {cont }}\right]$ / $\left[\mathrm{M}_{\mathrm{BBS} 1}-\mathrm{M}_{\text {cont }}\right]$; where $\mathrm{SD}$ indicates standard deviation and $\mathrm{M}$ indicates mean). The assay was redone for the plates with $Z^{\prime}$ less than 0.2 . These controls are included to ensure that assay performance complies with the established assay validation data package, which vigorously examines interplate, intraplate, and interassay variability per NIH-recommended large-scale screening guidelines (75).

$q P C R$ analyses. Total RNA was isolated following the standard TRIzol (Invitrogen) protocol. cDNA was synthesized from $1 \mu \mathrm{g}$ of total RNA using the QuantiTect kit (Qiagen). qPCR was performed with Power SYBR Green PCR Master Mix (Applied Biosystems) on a 7900HT (Applied Biosystems). Real-time data were collected and analyzed with a Sequence Detection System software package version 2.3 (Applied Biosystems). Primer sequences are listed in the Supplemental Table 3.

Zebrafish embryo manipulation and phenotyping. To determine the effect of bbs 4 or usp 38 suppression in zebrafish, we obtained previously (76) described MOs from Gene Tools (Gene Tools); bbs4-MO: 5'-CCGTTCTCATAGCGTCGTCCGCCA-3'; usp38-MO: 5'-CAAACAGAGTTTACTGACCTGTGGA-3'; zic1-MO (77): 5'-ACTGTGGTCCTGCGTCCAAGAGCAT-3'; ptma-MO: 5'-CCGAAGTTGTATTCATTACCTTTGC-3'; engase-MO: 5'-CTCATAAAAGGCGAAGTTACCTGAC-3'; $\quad d t x 1$-MO (78): 5'-TATCGACCCAGCTCACACAAGGGC-3'; tex36-MO: $\quad$ 5'-TTAAAGTGCGGTTATCTTACCCAGT-3'; $\quad$ tdrd12-MO (79): 5'-TGTATTTCCAGCATCTCCAATTTCT-3'; pitpnm2-MO: 5'-GTGTGTGCTCATACTGACCGTGTGC-3'; c14orf166-MO: 5'-TCAGTTCAGCCAAAATCTTACCTTT-3'; entpd6-MO: 5'-AATGTAAACATAC- 
CATTTGGCTCCA-3'; and enpp7-MO: 5'-TGCTGTTTTCCCTTTCTCCTTACCA-3'. We used the standard control MO purchased from Gene Tools as our negative control (5'-CCT CTT ACC TCA GTT ACA ATT TAT A-3'). This MO is also used to adjust the total amount of $\mathrm{MO}$ in the coinjection experiments. For example, in the experiment of Figure 2A, the injections was done as (a) control MO (1 ng), (b) bbs4-MO (0.5 ng) + control MO (0.5 ng), (c) bbs4-MO (0.5 ng) + usp38-MO (0.5 ng), and (d) control MO (0.5 ng) + usp38-MO (0.5 ng). The MO was injected into WT (EK x AB) zebrafish embryos at the 1- to 4-cell stage. CRISPR target sequences (bbs4: 5'-AGCTCCTGAGCTTCCCATCC-3' [60 pg], usp38: 5'-ACCCTGGAGAATAAGGCGGG-3' [7.5 pg]) were identified by crispr.mit.edu or CHOPCHOP (http://chopchop.cbu.uib.no) and in vitro synthesized with the GeneArt Precision gRNA synthesis kit (Invitrogen) following the manufacturer's manual. An injection solution of gRNA plus 100 pg Cas9 protein (PNA Bio) was injected into 1-cell stage WT zebrafish embryos. For CE phenotyping, embryos were initially scored as described previously (34) at the 8- to 10-somite stage, and images were captured using an AZ100 microscope and NIS Elements software (Nikon). The CE phenotypes were then quantitatively examined by measuring the anterior-posterior gap angle. Class I was defined by the angle larger than the mean angle of control embryos plus $2 \mathrm{SD}\left(\mathrm{M}_{\text {control }}+2 \mathrm{SD}\right)$, and Class II was defined by the angle larger than $\mathrm{M}_{\text {control }}+4 \mathrm{SD}$. To assess renal phenotypes, immunostaining with $\mathrm{Na} / \mathrm{K}$ ATPase antibody (1:20; DSHB) was performed in 4 dpf larvae as described (36). Stained larvae were imaged in glycerol using an AZ100 microscope (Nikon). Pairwise comparisons of bbs4 morphants or bbs4 F0 mutant embryos compared with cosuppression conditions consisting of $b b s 4$ plus usp 38 reagents for rescue efficacy were conducted using a $\chi^{2}$ test (GraphPad).

Immunoprecipitation and immunoblotting. HEK293 cells were cultured in DMEM containing 10\% HI-FBS and transfected with plasmids indicated in the figures. To assess the ubiquitination of $\beta$-cat, cells were treated with MG132 (Calbiochem) for 5 hours to prevent the degradation of ubiquitinated $\beta$-cat and then harvested with Subcellular Protein Fractionation Kit (Thermo Fisher Scientific). The lysates were incubated with $\beta$-cat (Santa Cruz Biotechnology Inc.; sc-7199) antibody at $4^{\circ} \mathrm{C}$ overnight, followed by incubation with the Protein G-coupled agarose beads (Santa Cruz Biotechnology Inc.) at $4^{\circ} \mathrm{C}$ for 2 hours. The beads were collected and washed with RIPA buffer (50 mM sodium chloride, $50 \mathrm{mM}$ Tris-HCl, $\mathrm{pH} 7.5$, $1 \%$ NP-40, 0.5\% sodium deoxycholate, and $0.1 \%$ SDS) (created in-house) with $1 \times$ proteasome inhibitor (Roche). Protein lysates were run on a $4 \%-15 \%$ SDS-PAGE gel (Bio-Rad) and immunoblotted with $\beta$-cat (1:3,000; Santa Cruz Biotechnology Inc., sc-7199) or ubiquitin (1:1,000; Abcam, ab8134) antibody. To assess the stability of NICD, transfected cells and tissue were lysed in RIPA buffer with $1 \times$ proteasome inhibitor (Roche) and centrifuged at $4^{\circ} \mathrm{C}$ for 15 minutes. Protein concentration was measured by Pierce BCA Protein assay Kit (Thermo Fisher Scientific) on the FLUOstar Omega microplate reader (BMG Labtech) and analyzed with MARS Data Analysis Software (BMG Labtech). Total protein lysates were separated by $4 \%-15 \%$ SDS-PAGE (Bio-Rad) with Spectra Multicolor Broad Range Protein Ladder (Fermentas) and transferred to Immuno-Blot PVDF Membrane (Bio-Rad). The membrane was blocked with 5\% nonfat milk and probed with commercial primary antibodies: anti-GAPDH (1:3000 Santa Cruz Biotechnology Inc., sc-32233) and anti-NICD (1:2000; Abcam, ab8925). Densitometric analysis was carried out with QuantityOne software (Bio-Rad).

Rhodopsin mislocalization assay. Zebrafish at $5 \mathrm{dpf}$ were fixed in $4 \%$ paraformaldehyde in PBS solution overnight at $4^{\circ} \mathrm{C}$. They were then submerged in $30 \%$ sucrose- $1 \times$ PBS for at least 24 hours at $4^{\circ} \mathrm{C}$ prior to embedding in OCT (Tissue-Tek) for transverse sectioning. Embryos were sectioned ( $\mu \mathrm{M}$-thick sections) along the transverse plane to attain retina sections at or near the optic nerve, which were then adhered onto slides for immunostaining.

Sections on slides were stored at $-20^{\circ} \mathrm{C}$ prior to staining. For staining, sections were initially washed with IF (immunofluorescence) buffer (1\% BSA, $1 \times$ PBST) for 10 minutes, followed by 30 minutes of incubation in blocking solution $(1 \times \mathrm{PBS}, 1 \%$ BSA, 10\% sheep serum) (Sigma-Aldrich). Sections were stained overnight at room temperature with rhodopsin antibody (1:1,000; EMD Millipore; 4D2) in blocking solution, followed incubation with a secondary antibody solution (Alexa 488 goat anti-mouse; Thermo Fisher Scientific, A32723) for 2 hours at room temperature. A final wash in IF buffer was performed prior to staining with DAPI (1:5,000; Roche) and mounted in Vectashield anti-fade medium (Vector Laboratories Inc.).

Fluorescent signals were visualized using Nikon 90i fluorescent microscope. In a normal retina, rhodopsin is localized to the outer segment and DAPI-positive cells outline the ONL vs. inner nuclear layer (INL) in the retina. To quantify the amount of rhodopsin mislocalization to the INL, we measured 
the total fluorescence of the rhodopsin signal in the DAPI-positive INL region of the retina (Image J). A background fluorescence value was taken from the ONL tissue, which was subtracted from the total rhodopsin fluorescence value of the INL to attain a corrected measure of rhodopsin mislocalization within the INL. Groups of 5-7 zebrafish were compared statistically by 1-way ANOVA with no posthoc correction (GraphPad).

Visual startle response (VSR). Automated VSR experiments were adapted from the protocol described in Scott et al. (55). Prior to the assay, $5 \mathrm{dpf}$ larvae were transferred to 96 -well plate and placed in the DanioVision (Noldus). The larvae went through an acclimation period (30 minutes, light) and a 2.5-minute testing period ( 5 cycles of 1 second dark followed by 29 seconds light). Ethovision software tracked and recorded the movement of zebrafish in 1-second increments for the entire duration of the experiment (32.5 minutes). The activity metrics were exported to excel file format. Using excel software, the activity results of the testing period were filtered out (Microsoft) and the number of VSR were determined at the threshold $>0.01$.

Statistics. GraphPad Prism for Windows (GraphPad Software) was used for statistical analyses. The quantification data are presented as the box-and-whiskers plot (Tukey's method). All data were analyzed using a 1-way ANOVA (retina staining and VSR), a 2-tailed Student's $t$ test (qPCR and Western blot quantification) or the $\chi^{2}$ test (CE and renal morphology). A $P$ value less than 0.05 was considered statistically significant.

Study approval. The zebrafish experiments were approved by the IRB (Duke University IACUC, Durham, North Carolina, USA). All animal work was performed in accordance with protocols approved by the Duke University IACUC.

\section{Author contributions}

NK and ICT designed the study, and ICT, KAA, JAT, OS, and PLT performed the experiments. NK and ICT wrote the main manuscript text, and ICT and KA prepared the figures. All authors approved and commented on the manuscript.

\section{Acknowledgments}

We thank Erica Davis and John Griffin for reading the manuscript and helpful comments. pLKO.1 TRC cloning vector was a gift from David Root (Addgene plasmid 10878). We are grateful to So Young Kim and all members in Duke Functional Genomics Shared Resource for their hospitality and guidance of RNAi screening. This work was supported by NIH grants R01HD042601, R01GM121317, R01DK072301, and P50DK096415 (NK) and by NRSA fellowship F32DK094578 (ICT). NK is a Distinguished George W. Brumley Professor.

Address correspondence to: Nicholas Katsanis, Stanley Manne Children's Research Institute, Ann \& Robert H. Lurie Children's Hospital of Chicago, 225 East Chicago Avenue, BOX 205, Chicago, Illinois 60611, USA. Phone: 312.503.7263; Email: nkatsanis@luriechildrens.org.

1. Gerdes JM, Davis EE, Katsanis N. The vertebrate primary cilium in development, homeostasis, and disease. Cell. 2009; 137(1):32-45.

2. Goetz SC, Anderson KV. The primary cilium: a signalling centre during vertebrate development. Nat Rev Genet. 2010;11(5):331-344

3. Seeley JV, Libby EM, Edwards KA, Seeley SK. Solvation parameter model of comprehensive two-dimensional gas chromatography separations. J Chromatogr A. 2009;1216(10):1650-1657.

4. Badano JL, Mitsuma N, Beales PL, Katsanis N. The ciliopathies: an emerging class of human genetic disorders. Annu Rev Genomics Hum Genet. 2006;7:125-148.

5. Baker K, Beales PL. Making sense of cilia in disease: the human ciliopathies. Am J Med Genet C Semin Med Genet. 2009;151C(4):281-295.

6. Zaghloul NA, Katsanis N. Mechanistic insights into Bardet-Biedl syndrome, a model ciliopathy. J Clin Invest. 2009;119(3):428-437.

7. Simons DL, Boye SL, Hauswirth WW, Wu SM. Gene therapy prevents photoreceptor death and preserves retinal function in a Bardet-Biedl syndrome mouse model. Proc Natl Acad Sci USA. 2011;108(15):6276-6281.

8. Reiter JF, Leroux MR. Genes and molecular pathways underpinning ciliopathies. Nat Rev Mol Cell Biol. 2017;18(9):533-547.

9. Dean M, et al. Genetic restriction of HIV-1 infection and progression to AIDS by a deletion allele of the CKR5 structural gene. Hemophilia Growth and Development Study, Multicenter AIDS Cohort Study, Multicenter Hemophilia Cohort Study, San Francisco City Cohort, ALIVE Study. Science. 1996;273(5283):1856-1862.

10. Cohen J, Pertsemlidis A, Kotowski IK, Graham R, Garcia CK, Hobbs HH. Low LDL cholesterol in individuals of African descent resulting from frequent nonsense mutations in PCSK9. Nat Genet. 2005;37(2):161-165.

11. Cohen JC, Boerwinkle E, Mosley TH, Hobbs HH. Sequence variations in PCSK9, low LDL, and protection against coronary heart disease. NEngl J Med. 2006;354(12):1264-1272. 
12. Temel RE, et al. Hepatic Niemann-Pick C1-like 1 regulates biliary cholesterol concentration and is a target of ezetimibe. JClin Invest. 2007;117(7):1968-1978.

13. Myocardial Infarction Genetics Consortium Investigators, et al. Inactivating mutations in NPC1L1 and protection from coronary heart disease. $N$ Engl J Med. 2014;371(22):2072-2082.

14. Flannick J, et al. Loss-of-function mutations in SLC30A8 protect against type 2 diabetes. Nat Genet. 2014;46(4):357-363.

15. Buchovecky CM, et al. A suppressor screen in Mecp2 mutant mice implicates cholesterol metabolism in Rett syndrome. Nat Genet. 2013;45(9):1013-1020.

16. Armakola M, et al. Inhibition of RNA lariat debranching enzyme suppresses TDP-43 toxicity in ALS disease models. Nat Genet. 2012;44(12):1302-1309.

17. Kramer NJ, et al. CRISPR-Cas9 screens in human cells and primary neurons identify modifiers of C9ORF72 dipeptide-repeat-protein toxicity. Nat Genet. 2018;50(4):603-612.

18. Nauli SM, et al. Polycystins 1 and 2 mediate mechanosensation in the primary cilium of kidney cells. Nat Genet. 2003;33(2):129-137.

19. Ramamurthy V, Cayouette M. Development and disease of the photoreceptor cilium. Clin Genet. 2009;76(2):137-145.

20. Liu YP, et al. Ciliopathy proteins regulate paracrine signaling by modulating proteasomal degradation of mediators. $J$ Clin Invest. 2014;124(5):2059-2070.

21. Kim JC, et al. The Bardet-Biedl protein BBS4 targets cargo to the pericentriolar region and is required for microtubule anchoring and cell cycle progression. Nat Genet. 2004;36(5):462-470.

22. Mykytyn K, et al. Identification of the gene that, when mutated, causes the human obesity syndrome BBS4. Nat Genet. 2001;28(2):188-191.

23. Gerdes JM, et al. Disruption of the basal body compromises proteasomal function and perturbs intracellular Wnt response. Nat Genet. 2007;39(11):1350-1360

24. Corbit KC, et al. Kif3a constrains beta-catenin-dependent Wnt signalling through dual ciliary and non-ciliary mechanisms. Nat Cell Biol. 2008;10(1):70-76.

25. Barrows NJ, Le Sommer C, Garcia-Blanco MA, Pearson JL. Factors affecting reproducibility between genome-scale siRNA-based screens. J Biomol Screen. 2010;15(7):735-747.

26. Leung JY, et al. Activation of AXIN2 expression by beta-catenin-T cell factor. A feedback repressor pathway regulating Wnt signaling. J Biol Chem. 2002;277(24):21657-21665.

27. Yan D, et al. Elevated expression of axin 2 and hnkd mRNA provides evidence that Wnt/beta -catenin signaling is activated in human colon tumors. Proc Natl Acad Sci USA. 2001;98(26):14973-14978.

28. Simons M, et al. Inversin, the gene product mutated in nephronophthisis type II, functions as a molecular switch between Wnt signaling pathways. Nat Genet. 2005;37(5):537-543.

29. Wheway G, et al. An siRNA-based functional genomics screen for the identification of regulators of ciliogenesis and ciliopathy genes. Nat Cell Biol. 2015;17(8):1074-1087.

30. Xie Y. Structure, assembly and homeostatic regulation of the 26S proteasome. J Mol Cell Biol. 2010;2(6):308-317.

31. Park J, Kwon MS, Kim EE, Lee H, Song EJ. USP35 regulates mitotic progression by modulating the stability of Aurora B. Nat Commun. 2018;9(1):688.

32. Wang Y, et al. Deubiquitinating enzymes regulate PARK2-mediated mitophagy. Autophagy. 2015;11(4):595-606.

33. Clague MJ, Barsukov I, Coulson JM, Liu H, Rigden DJ, Urbé S. Deubiquitylases from genes to organism. Physiol Rev. 2013;93(3):1289-1315

34. Leitch CC, et al. Hypomorphic mutations in syndromic encephalocele genes are associated with Bardet-Biedl syndrome. Nat Genet. 2008;40(4):443-448.

35. Imhoff O, et al. Bardet-Biedl syndrome: a study of the renal and cardiovascular phenotypes in a French cohort. Clin J Am Soc Nephrol. 2011;6(1):22-29.

36. Lindstrand A, et al. Recurrent CNVs and SNVs at the NPHP1 locus contribute pathogenic alleles to Bardet-Biedl syndrome. Am J Hum Genet. 2014;94(5):745-754.

37. Vasilyev A, et al. Collective cell migration drives morphogenesis of the kidney nephron. PLoS Biol. 2009;7(1):e9.

38. Stainier DYR, et al. Guidelines for morpholino use in zebrafish. PLoS Genet. 2017;13(10):e1007000.

39. Mooney MR, Davis EE, Katsanis N. Analysis of Single Nucleotide Variants in CRISPR-Cas9 Edited Zebrafish Exomes Shows No Evidence of Off-Target Inflation [published online ahead of print October 11, 2019]. Fron Genet. doi: 10.3389/fgene.2019.00949.

40. Gerhardt C, Wiegering A, Leu T, Rüther U. Control of Hedgehog Signalling by the Cilia-Regulated Proteasome. J Dev Biol. 2016;4(3):E27.

41. Spence JR, Madhavan M, Ewing JD, Jones DK, Lehman BM, Del Rio-Tsonis K. The hedgehog pathway is a modulator of retina regeneration. Development. 2004;131(18):4607-4621.

42. Bigelow RL, et al. Transcriptional regulation of bcl-2 mediated by the sonic hedgehog signaling pathway through gli-1. $J$ Biol Chem. 2004;279(2):1197-1205

43. Johnson RL, et al. Human homolog of patched, a candidate gene for the basal cell nevus syndrome. Science. 1996;272(5268):1668-1671.

44. Boselli M, et al. An inhibitor of the proteasomal deubiquitinating enzyme USP14 induces tau elimination in cultured neurons. J Biol Chem. 2017;292(47):19209-19225.

45. Lee BH, et al. Enhancement of proteasome activity by a small-molecule inhibitor of USP14. Nature. 2010;467(7312):179-184

46. Bek T, Rosenberg T. Clinical pathology and retinal vascular structure in the Bardet-Biedl syndrome. Br J Ophthalmol. 1995;79(1):76-80.

47. Campo RV, Aaberg TM. Ocular and systemic manifestations of the Bardet-Biedl syndrome. Am J Ophthalmol. 1982;94(6):750-756.

48. Gerth C, Zawadzki RJ, Werner JS, Héon E. Retinal morphology in patients with BBS1 and BBS10 related Bardet-Biedl Syndrome evaluated by Fourier-domain optical coherence tomography. Vision Res. 2008;48(3):392-399.

49. Runge P, Calver D, Marshall J, Taylor D. Histopathology of mitochondrial cytopathy and the Laurence-Moon-Biedl syndrome. Br J Ophthalmol. 1986;70(10):782-796. 
50. Beales PL, et al. IFT80, which encodes a conserved intraflagellar transport protein, is mutated in Jeune asphyxiating thoracic dystrophy. Nat Genet. 2007;39(6):727-729.

51. Khanna H, et al. A common allele in RPGRIP1L is a modifier of retinal degeneration in ciliopathies. Nat Genet. 2009;41(6):739-745.

52. Parisi MA. Clinical and molecular features of Joubert syndrome and related disorders. Am J Med Genet C Semin Med Genet. 2009;151C(4):326-340.

53. Abd-El-Barr MM, et al. Impaired photoreceptor protein transport and synaptic transmission in a mouse model of Bardet-Biedl syndrome. Vision Res. 2007;47(27):3394-3407.

54. Lobanova ES, et al. Increased proteasomal activity supports photoreceptor survival in inherited retinal degeneration. Nat Com mun. 2018;9(1):1738.

55. Scott CA, Marsden AN, Slusarski DC. Automated, high-throughput, in vivo analysis of visual function using the zebrafish. Dev Dyn. 2016;245(5):605-613.

56. Pazour GJ, et al. The intraflagellar transport protein, IFT88, is essential for vertebrate photoreceptor assembly and maintenance. J Cell Biol. 2002;157(1):103-113.

57. Yoder BK, Hou X, Guay-Woodford LM. The polycystic kidney disease proteins, polycystin-1, polycystin-2, polaris, and cystin are co-localized in renal cilia. J Am Soc Nephrol. 2002;13(10):2508-2516.

58. McIntyre JC, et al. Gene therapy rescues cilia defects and restores olfactory function in a mammalian ciliopathy model. Nat Med. 2012;18(9):1423-1428.

59. Sukumaran S, Perkins BD. Early defects in photoreceptor outer segment morphogenesis in zebrafish ift57, ift88 and ift172 Intraflagellar Transport mutants. Vision Res. 2009;49(4):479-489.

60. Twigg SR, et al. Gain-of-Function Mutations in ZIC1 Are Associated with Coronal Craniosynostosis and Learning Disability. Am J Hum Genet. 2015;97(3):378-388.

61. Jiang X, et al. Distinctive roles of PHAP proteins and prothymosin-alpha in a death regulatory pathway. Science. 2003;299(5604):223-226.

62. Hsiao HW, et al. Deltex1 is a target of the transcription factor NFAT that promotes T cell anergy. Immunity. 2009;31(1):72-83

63. Mukherjee A, Veraksa A, Bauer A, Rosse C, Camonis J, Artavanis-Tsakonas S. Regulation of Notch signalling by non-visual beta-arrestin. Nat Cell Biol. 2005;7(12):1191-1201.

64. Zheng L, Conner SD. PI5P4K $\gamma$ functions in DTX1-mediated Notch signaling. Proc Natl Acad Sci USA. 2018;115(9):E1983-E1990.

65. Tian G, Ropelewski P, Nemet I, Lee R, Lodowski KH, Imanishi Y. An unconventional secretory pathway mediates the cilia targeting of peripherin/rds. J Neurosci. 2014;34(3):992-1006.

66. Lev S, Hernandez J, Martinez R, Chen A, Plowman G, Schlessinger J. Identification of a novel family of targets of PYK2 related to Drosophila retinal degeneration B (rdgB) protein. Mol Cell Biol. 1999;19(3):2278-2288.

67. Chang MY, et al. Haploinsufficiency of $\mathrm{Pkd} 2$ is associated with increased tubular cell proliferation and interstitial fibrosis in two murine Pkd2 models. Nephrol Dial Transplant. 2006;21(8):2078-2084

68. Nadasdy T, Laszik Z, Lajoie G, Blick KE, Wheeler DE, Silva FG. Proliferative activity of cyst epithelium in human renal cystic diseases. J Am Soc Nephrol. 1995;5(7):1462-1468.

69. Lee K, Battini L, Gusella GL. Cilium, centrosome and cell cycle regulation in polycystic kidney disease. Biochim Biophys Acta. 2011;1812(10):1263-1271.

70. Bangs F, Anderson KV. Primary Cilia and Mammalian Hedgehog Signaling. Cold Spring Harb Perspect Biol. 2017 ;9(5):a028175.

71. Breslow DK, et al. A CRISPR-based screen for Hedgehog signaling provides insights into ciliary function and ciliopathies. Nat Genet. 2018;50(3):460-471.

72. Zhang Y, et al. USP44 regulates centrosome positioning to prevent aneuploidy and suppress tumorigenesis. J Clin Invest. 2012;122(12):4362-4374.

73. Wang W, Wu T, Kirschner MW. The master cell cycle regulator APC-Cdc20 regulates ciliary length and disassembly of the primary cilium. Elife. 2014;3:e03083.

74. Veeman MT, Slusarski DC, Kaykas A, Louie SH, Moon RT. Zebrafish prickle, a modulator of noncanonical Wnt/Fz signaling, regulates gastrulation movements. Curr Biol. 2003;13(8):680-685.

75. Iversen PW, et al. HTS Assay Validation. In: Sittampalam GS, et al, eds. Assay Guidance Manual. Bethesda, Maryland, USA: Eli Lilly \& Company, National Center for Advancing Translational Sciences; 2004

76. Bögershausen N, et al. RAP1-mediated MEK/ERK pathway defects in Kabuki syndrome. J Clin Invest. 2015;125(9):3585-3599.

77. Elsen GE, Choi LY, Millen KJ, Grinblat Y, Prince VE. Zic1 and Zic4 regulate zebrafish roof plate specification and hindbrain ventricle morphogenesis. Dev Biol. 2008;314(2):376-392.

78. Hsieh FY, et al. Dner inhibits neural progenitor proliferation and induces neuronal and glial differentiation in zebrafish. Dev Biol. 2013;375(1):1-12.

79. Huang HT, et al. A network of epigenetic regulators guides developmental haematopoiesis in vivo. Nat Cell Biol. 2013;15(12):1516-1525. 